\title{
Atrial remodeling in atrial fibrillation and association between microRNA network and atrial fibrillation
}

\author{
ZHANG Ying, DONG DeLi \& YANG BaoFeng* \\ Department of Pharmacology, State-Province Key Laboratories of Biomedicine-Pharmaceutics of China, Key Laboratory of \\ Cardiovascular Research, Ministry of Education, Harbin 150086, China
}

Received June 22, 2011; accepted August 24, 2011

\begin{abstract}
Atrial fibrillation $(\mathrm{AF})$ remains one of the leading causes of morbidity and mortality in the world which are related to palpitations, fainting, congestive heart failure or stroke. The mechanism for atrial fibrillation has been identified as electrical remodeling, structure remodeling and intracellular calcium handling remodeling. microRNAs (miRNAs) have recently emerged as one of the important factors in regulating gene expression. So far, thousands of miRNA genes have been found in diverse animals with the function of regulating cell death, cell proliferation, haematopoiesis and even participate in the processing of cardiovascular disease. In this review, we summarize the mechanism of AF and the association of microRNAs network with AF. We provide a potential perspective of miRNAs as the therapeutic target for AF.
\end{abstract}

atrial fibrillation, remodeling, microRNA

Citation: Zhang Y, Dong D L, Yang B F. Atrial remodeling in atrial fibrillation and association between microRNA network and atrial fibrillation. Sci China Life Sci, 2011, 54: 1097-1102, doi: 10.1007/s11427-011-4241-3

Atrial fibrillation (AF) is the most common type of cardiac arrhythmia in clinical practice and is highly age dependent. Its prevalence ranges from $0.5 \%$ at $50-59$ years old to almost $9 \%$ at $80-89$ years old [1]. AF is often underestimated because it is usually asymptomatic and is not life-threatening. However, it can induce palpitations, fainting, congestive heart failure, or stroke due to electrical disturbance, as well as thromboembolism, all of which are associated with high morbidity and mortality [2]. Further research into the predisposing factors and precautionary measures, and the development of more efficient therapeutic tools are needed to reduce these substantial lifetime risks. Although traditional drugs are helpful for maintaining normal sinus rhythm, they are associated with numerous side effects such as nausea, dizziness, fatigue and even ventricular arrhythmias, while non-pharmacological therapies may lead to complications such as infection and thrombosis. It is therefore important to

*Corresponding author (email: yangbf@ems.hrbmu.edu.cn) understand the mechanism responsible for $\mathrm{AF}$ and to develop new methods of treatment.

This review summarizes the mechanism of AF and its association with the microRNA (miRNA) network, thus providing a potentially useful perspective of miRNAs as a therapeutic target for AF.

\section{Mechanisms of AF}

AF can be initiated and maintained by ectopic foci or by single- or multiple-circuit re-entry induced by a variety of substrates such as high blood pressure, coronary artery disease, heart attack, and hyperthyroidism [2]. Sustained AF has been suggested to result from series of remodeling events within the atria (Table 1, Figure 1). Distinct changes in atrial tachycardia-induced remodeling shorten the atrial effective refractory period (ERP) and action potential duration (APD), and reduce its physiological rate adaptation as a 
Table 1 Electrical remodeling of atrial fibrillation

\begin{tabular}{|c|c|c|c|c|c|}
\hline Ion channels & Gene & Currents & $\begin{array}{c}\text { Change of current } \\
\text { level }\end{array}$ & $\begin{array}{c}\text { Change of protein } \\
\text { level }\end{array}$ & $\begin{array}{c}\text { Change of mRNA } \\
\text { level }\end{array}$ \\
\hline L-type calcium channel (Cav1.2) & CACNAIC & $I_{\mathrm{Ca}-\mathrm{L}}$ & $\downarrow$ & $\downarrow$ or - & $\downarrow$ or - \\
\hline Inward rectifier potassium channel (Kir2.1) & KCNJ2 & $I_{\mathrm{K} 1}$ & $\uparrow$ & - & - \\
\hline Transient outward potassium channel (Kv4.3) & $K C N D 3$ & $I_{\mathrm{to}}$ & $\downarrow$ & $\downarrow$ & $\downarrow$ \\
\hline Ultrarapid delayed rectifier potassium channel (Kv1.5) & KCNA5 & $I_{\text {Kur }}$ & $\downarrow$ & $\downarrow$ & $\downarrow$ \\
\hline Acetylcholine-activated potassium channel (Kir3.1) & $K C N J 3$ & $I_{\mathrm{K}-\mathrm{ACh}}$ & $\downarrow$ & $\downarrow$ & $\downarrow$ \\
\hline ATP-sensitive potassium channel (Kir6.2) & KCNJ11 & $I_{\mathrm{K}-\mathrm{ATP}}$ & $\downarrow$ & $\downarrow$ & $\downarrow$ \\
\hline Fast sodium channel (Nav1.5) & $S C N 5 A$ & $I_{\mathrm{Na}}$ & $\downarrow$ & - & - \\
\hline
\end{tabular}

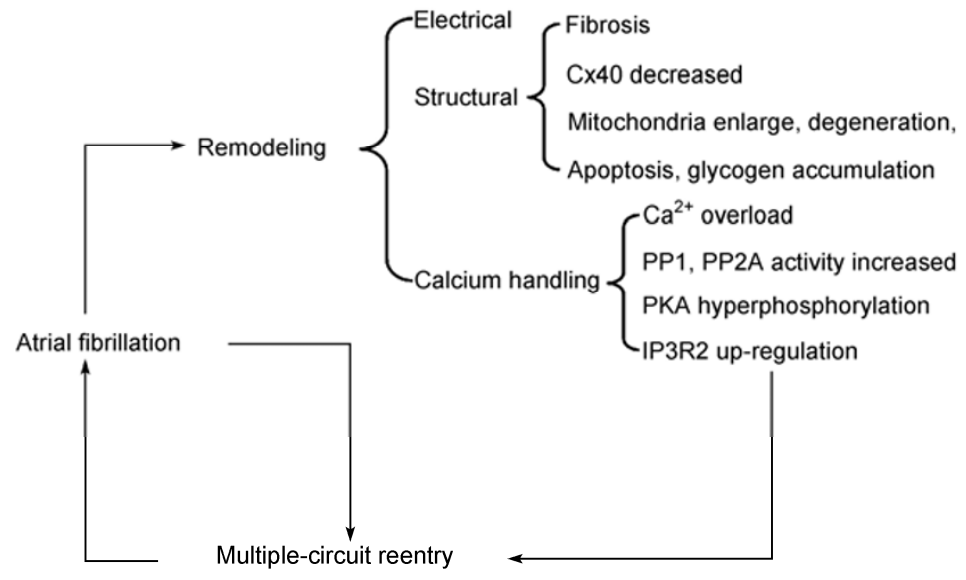

Figure 1 Atrial remodeling in atrial fibrillation. PP1, type 1 phosphatase; PP2A, type 2A phosphatase; PKA, protein kinase A.

result of ion-channel remodeling and abnormal $\mathrm{Ca}^{2+}$ handling.

\subsection{Electrical remodeling}

Ion-channel remodeling involves changes in current density or in ion channel mRNA or protein expression. Electrophysiological studies have revealed a series of current changes in AF, including changes in L-type $\mathrm{Ca}^{2+}\left(I_{\mathrm{Ca}-\mathrm{L}}\right)$, inward rectifier potassium $\left(I_{\mathrm{K} 1}\right)$, transient outward potassium $\left(I_{\mathrm{to}}\right)$, ultra-rapid delayed rectifier potassium $\left(I_{\mathrm{Kur}}\right)$, acetylcholine-activated potassium $\left(I_{\mathrm{K}-\mathrm{ACh}}\right)$, ATP-sensitive potassium $\left(I_{\mathrm{K}-\mathrm{ATP}}\right)$, and fast sodium $\left(I_{\mathrm{Na}}\right)$ currents.

$I_{\mathrm{Ca}-\mathrm{L}}$ decreases and the inactivation is slowed in both the canine pacing model of AF and in chronic AF patients [3]. Increased atrial rate could cause cellular $\mathrm{Ca}^{2+}$ overload as a result of $\mathrm{Ca}^{2+}$ intake [4], and reduction in $I_{\mathrm{Ca}-\mathrm{L}}$ can therefore decrease the $\mathrm{Ca}^{2+}$ entry and protect against the harmful effects of progressive $\mathrm{Ca}^{2+}$ overload. However, decreased $I_{\mathrm{Ca}-\mathrm{L}}$ can further promote and maintain AF by reducing APD and APD adaptation to rate [5]. However, changes in $I_{\mathrm{Ca}-\mathrm{L}}$ alone are insufficient to account for the reduction in APD, and increased $I_{\mathrm{K} 1}$ has a greater influence on the reduction of atrial APD in humans. At hyperpolarizing potentials, $I_{\mathrm{K} 1}$ has been shown to be increased in appendages and left atrial myocytes in AF patients, but not in a canine fast-pacing model [6].

$I_{\text {to }}$ is thought to increase in atrial myocytes isolated from patients with chronic AF, to sustain ERP and APD reduction. However, the opposite effect is seen in myocytes isolated from both the left and right atrial appendages of patients in chronic AF, suggesting that decreased $I_{\text {to }}$ is the result, rather than the cause, of chronic AF [6].

$I_{\text {Kur }}$ plays a significant role in atrial, but not in ventricular repolarization and has therefore been considered as a target for "atrial-selective" anti-arrhythmic drugs [7]. However, recent research has determined that the $I_{\text {Kur }}$ amplitude is significantly reduced and its current density is lower in $\mathrm{AF}$ patients, compared to sinus rhythm patients [8]. These changes may abbreviate APD and ERP.

Abnormal parasympathetic activation has been shown to predispose the atria to fibrillation [9]. Acetylcholine released from the vagus nerve after parasympathetic stimulation binds muscarinic receptors to activate the muscarinic-gated potassium channel $I_{\mathrm{K}-\mathrm{Ach}}$. Continuous activation of $I_{\mathrm{K}-\mathrm{ACh}}$ may aggravate the abbreviation of APD and increase AF vulnerability [10]. Furthermore, in order to adapt to the high beating rate, $I_{\mathrm{K}-\mathrm{ACh}}$ is reduced to counteract the shortening of APD [11].

The ATP-sensitive potassium channel is associated with excitability and metabolic status. The increase in $I_{\mathrm{K}-\mathrm{ATP}}$ amplitude after treatment with the $I_{\mathrm{K} \text {-ATP }}$ channel opener rilmakalim was smaller in human atrial AF myocytes than in those from a sinus-rhythm group, suggesting that $I_{\mathrm{K} \text {-ATP }}$ is down-regulated during chronic AF [12].

$I_{\mathrm{Na}}$ is a major determinant of conduction velocity. Rapid 
atrial rates can significantly reduce both $I_{\mathrm{Na}}$ density and conduction velocity, which can trigger AF [13]. In contrast, voltage-dependent inactivation of $I_{\mathrm{Na}}$ is shifted to more positive potentials by AF in humans. Electrical remodeling of other channels such as $I_{\mathrm{Kr}}, I_{\mathrm{Ks}}$, and $I_{\mathrm{f}}$ has not been well established [14].

Changes in ion channels at the gene or protein expression level can account for the generation and progression of AF, and reductions of L-type $\mathrm{Ca}^{2+}$ channel (Cav1.2) mRNA and protein are an important feature in clinical and animal experimental studies [15]. These changes might involve calpain, which is activated by increased cytoplasmic $\mathrm{Ca}^{2+}$ to degrade L-type $\mathrm{Ca}^{2+}$ channels [16]. However, these changes in mRNA or protein expression in $\mathrm{AF}$ are inconclusive. Some researchers have reported conflicting results concerning Cav1.2 mRNA and protein expression [17,18], and further research into Cav1.2 mRNA and protein changes at the molecular level are needed. Both mRNA and protein levels of the transient outward channel (Kv4.3), the ATPsensitive potassium channel (Kir6.2), the acetylcholinedependent potassium channel (Kir3.4) and the ultra-rapid delayed rectifier potassium channel (Kv1.5) are decreased in AF patients [19-21]. These decreased potassium channel expressions help to counteract the rapid atrial rate [20]. In contrast, no change in the mRNA or protein levels of the inward rectifier $\mathrm{K}^{+}$channel (Kir2.1) is seen. Researchers have also found that mutations in SCN5A and KCNA5 are a major cause of familial AF [11].

\subsection{Structural remodeling}

In addition to electrical remodeling, dramatic structural remodeling occurs in AF cardiomyocytes, and fibrosis, as a hallmark of arrhythmogenic structural remodeling, has been detected in different models of AF and in patients with AF. Canine models with aging, mitral regurgitation, or atrial or ventricular tachypacing all have $\mathrm{AF}$ and obvious atrial fibrosis, indicating a clinical relationship between atrial fibrosis and AF [22]. Fibrosis can disrupt cardiac conduction by affecting intermyocyte coupling, as well as other factors, such as angiotensin II and transforming growth factor- $\beta 1$ (TGF- $\beta 1$ ), which are regarded as profibrotic factors [23].

Cardiac impulse conduction depends on gap junction channels (composed of $\mathrm{Cx} 43$ and $\mathrm{Cx} 40$ ), which are closely associated with cell-to-cell adhesion and mechanical coupling [24]. Cx40 expression decreases and the protein becomes more heterogeneously distributed after long periods of fast pacing, and the localized conduction abnormalities contribute to initiation and self-perpetuation of re-entry pathways and AF [25]. Further ultrastructural changes in atrial myocytes have been observed in different models of chronic AF and in AF patients, including enlarged nuclei with dispersed chromatin, increased number and size of mitochondria, degeneration and apoptosis of atrial myocytes, increased cell size, accumulation of glycogen, and disrup- tion of the sarcoplasmic reticulum (SR) [26]. Structural remodeling thus appears to play an important role in the perpetuation of $\mathrm{AF}$.

\subsection{Intracellular $\mathrm{Ca}^{2+}$ handling remodeling}

Abnormal intracellular $\mathrm{Ca}^{2+}$ handling is an important aspect of AF pathophysiology and might contribute to the initiation and perpetuation of $\mathrm{AF}$, but also develops as a result of AF. Intracellular $\mathrm{Ca}^{2+}$ overload induces arrhythmogenesis by promoting focal firing and atrial electrical remodeling during AF. The molecular basis of abnormal $\mathrm{Ca}^{2+}$ handling in AF is complex and relies on several phosphorylation processes. (i) Increased type 1 and type $2 \mathrm{~A}$ phosphatase activities can reduce $I_{\mathrm{Ca}-\mathrm{L}}$ amplitude by enhancing channel dephosphorylation in human AF. (ii) Protein kinase A hyperphosphorylation of the cardiac ryanodine receptor can induce $\mathrm{Ca}^{2+}$ leak from the SR, contributing to intracellular $\mathrm{Ca}^{2+}$ overload in AF [4]. (iii) Up-regulation of IP3 receptor (IP3R2) expression aggravates $\mathrm{Ca}^{2+}$ release from the $\mathrm{SR}$, and $\mathrm{Ca}^{2+}$-induced $\mathrm{Ca}^{2+}$ release in turn elevates IP3R2 expression.

\section{2 miRNA biology}

miRNAs are small RNAs (about 22 nucleotides) that regulate the expression of complementary mRNAs as 'micromanagers of gene expression'. They are associated with the RNA-induced silencing complex through partly complementary sequences localized in the $3^{\prime}$ untranslated region of the mRNA target [27]. Moreover, some miRNAs occasionally induce changes in chromatin structure of the targeted promoter, e.g., by decreasing active-state histone modifications, or increasing histone methylation marks and DNA methylation [28]. Recent studies have shown significant polymorphisms in miRNAs. Unlike polymorphisms in coding genes, polymorphisms in miRNAs are closely related to various diseases, including tumors, nervous system diseases, and cardiovascular diseases. To date, thousands of miRNA genes have been found in diverse animals, with functions in regulating cell death, cell proliferation, and hematopoiesis, and even in the process of cardiovascular disease [29,30]. Increasing evidence has identified an important role for miRNAs in AF initiation and maintenance.

\section{Role of miRNAs in AF initiation and maintenance}

\subsection{Effect of miRNAs on electrical and molecular re- modeling in AF}

Our recent research found that miR-328 contributes to the adverse atrial electric remodeling in AF through targeting 
L-type $\mathrm{Ca}^{2+}$ channel genes. Levels of miR-328 were elevated by 3.5-3.9-fold in left atrial samples from dogs with model AF and in AF patients, detected using microarray and real-time polymerase chain reaction. Furthermore CACNA1C and CACNB1, which encode the cardiac L-type $\mathrm{Ca}^{2+}$ channel $\alpha 1 \mathrm{c}-$ and $\beta 1$ subunits, are predicted to be potential targets of miR-328. This hypothesis has been verified using adenovirus infection and a transgenic approach to force the expression of miR-328, resulting in enhanced AF vulnerability, diminished L-type $\mathrm{Ca}^{2+}$ currents, and shortened atrial APD [29].

Girmatsion et al. identified the involvement of another miRNA in the maintenance of AF [31]. They found that $I_{\mathrm{K} 1}$ density, and Kir2.1 mRNA and protein expression were increased in tissue samples from AF patients. Expression of inhibitory miR-1 was reduced by approximately $86 \%$ in tissue samples from AF patients. Regulation of Kir2.1 by miR-1 was verified by in vitro experiments. This research demonstrated that miR-1 levels were greatly reduced in AF patients, possibly contributing to the up-regulation of Kir2.1 subunits and leading to increased $I_{\mathrm{K} 1}$, which is an important factor in AF maintenance.

In addition to the roles of miR-328 and miR-1 in the process of AF, Xiao et al. investigated another miRNA signature in AF with mitral stenosis (MS). They revealed that 28 miRNAs were differentially expressed in MS patients with AF compared to those without AF, of which miR-1202 was the most dysregulated. The distinct miRNA expression signature of AF with MS can therefore aid in the development of therapeutic interventions based on rational target selection in these patients [32].

\subsection{Effect of miRNAs on structural remodeling in AF}

In addition to electrical and molecular remodeling, miRNAs also promote structural remodeling in AF. We demonstrated that nicotine produced significant down-regulation of miR-133 and miR-590, which alleviated the repression of their target genes in TGF- $\beta 1$ and TGF- $\beta$ receptor type II protein expression. Ex vivo experiments verified that transfection of miR-133 and miR-590 into cultured atrial fibroblasts decreased TGF- $\beta 1$ and TGF- $\beta$ receptor type II expression, as well as collagen content. These effects were abolished by antisense oligonucleotides against miR-133 or miR-590. The profibrotic response to nicotine in the canine atrium was thus critically dependent upon down-regulation of the antifibrotic miRNAs miR-133 and miR-590 [33].

Other miRNAs have been reported to regulate myocardial fibrosis, including the miR-29 family, miR-30, and miR-208. The miR-29 family can regulate a series of target genes that encode proteins participating in fibrosis, such as multiple collagens, fibrillins, and elastin. Down-regulation of miR-29 has been shown to depress the expression of these mRNAs and enhance the fibrotic response [34]. Connective tissue growth factor is a key profibrotic protein that can be directly down-regulated by both miR-133 and miR-30. Restoring these miRNAs to normal levels thus has the potential to control the structural changes in the extracellular matrix of the myocardium [35]. miR-208 is encoded by an intron of the $\alpha \mathrm{MHC}$ gene and regulates $\alpha \mathrm{MHC}$ gene expression in response to stress and hormonal signaling. This regulation plays an important role in cardiomyocyte hypertrophy and fibrosis [36] (Figure 2).

\section{Modulation of miRNAs by transcription fac- tors}

miRNA alterations in AF have been associated with transcription factors. mRNA and protein levels of the transcrip-

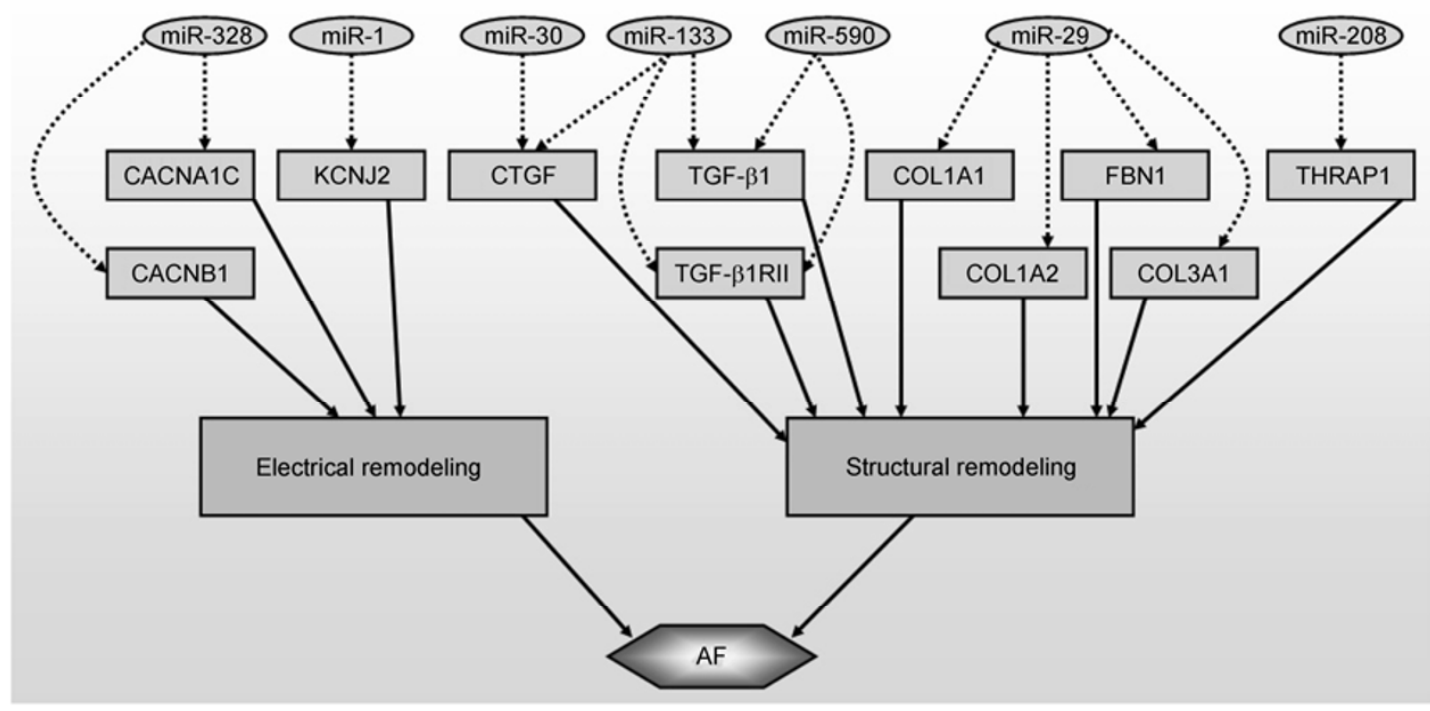

Figure 2 Electrical and structural remodeling mechanisms in atrial fibrillation regulated by miRNAs. CTGF, connective tissue growth factor; FBN, fibrillin; THRAP, thyroid hormone receptor associated protein; COL, collagen. 
tion factor nuclear factor $\kappa-\mathrm{B}(\mathrm{NF}-\kappa \mathrm{B})$ were increased in an atrial tachypacing canine model. Two research groups identified an accumulation of NF- $\kappa \mathrm{B}$ in human atrial tissue slices subjected to rapid pacing for $24 \mathrm{~h}$, as well as in AF patients [37]. We verified that enhancement of NF- $\mathrm{B}$ up-regulated miR-328 transcription, using a chromatin immunoprecipitation assay. Other aspects of miRNA regulation have been revealed by different research groups. For example, transcription of miR-145 and miR-143 can be regulated by serum response factor, myocardin, and by Nkx2-5 (NK2 transcription factor related, locus 5), while miR-145 and miR-143 can also feed back to a network of transcription factors, including Klf4 (Kruppel-like factor 4), myocardin and Elk-1 (ELK1, member of the ETS oncogene family) [38]. Schlesinger et al. [39] have mapped the Srf-centered transcription network, integrating Srf-binding events, H3ac, miRNAs, and differential expression in Srf knockdown.

\section{Clinical uses of miRNAs}

Many studies have confirmed the involvement of miRNAs in cardiac diseases, especially in AF, but the potential to translate the results of this basic research into clinical practice remains to be determined. Initial studies have detected plasma miRNA levels and investigated the possibility of gene therapy in an animal model of acute myocardial infarction (AMI). In addition, miR-1 levels were found to be significantly higher in the plasma of AMI patients, compared to non-AMI subjects, suggesting that circulating miR-1 may be a novel independent biomarker for the diagnosis of AMI [40].

We recently used miRNA mimics and a modified anti-miRNA antisense inhibitor technique to interfere with the expression of target genes. These approaches may represent a plausible strategy for miRNA therapy of human diseases. miRNA mimics are gene-specific and contain 7-8 nucleotide seed sequences that match the target gene completely. This technique can increase the binding efficiency and specificity of miRNAs to their target genes, thus avoiding off-target effect. Multiple anti-miRNA antisense inhibitors involve the engineering of multiple antisense units into a single unit, which is able to simultaneously silence multiple target miRNAs. This technique allows the rescue of multiple miRNAs that are affected in human diseases. These studies suggest that miRNA mimics and multiple anti-miRNA antisense inhibitors have the potential to rescue miRNA imbalances occurring in AF.

Our preliminary studies have shown that correcting miR-328 levels to normal using antagomiR reversed the AF conditions, while genetic knockdown of endogenous miR-328 decreased AF vulnerability. Antisense miR-328 thus shows great potential for the treatment of AF. Further research into the networks of miRNAs is likely to reveal the potential to cure more human diseases, though considerable work remains to be done.

This work was supported by the National Natural Science Foundation of China (Grant No. 81100122) to Zhang Ying, China Postdoctoral Special Science Foundation to Dong DeLi, and National Basic Research Program of China (Grant Nos. 2007CB512000 and 2007CB512006) to Yang BaoFeng.

1 Kannel W B, Wolf P A, Benjamin E J, et al. Prevalence, incidence, prognosis, and predisposing conditions for atrial fibrillation: population-based estimates. Am J Cardiol, 1998, 82: 2N-9N

2 Nattel S. New ideas about atrial fibrillation 50 years on. Nature, 2002, 415: 219-226

3 Bosch R F, Zeng X, Grammer J B, et al. Ionic mechanisms of electrical remodeling in human atrial fibrillation. Cardiovasc Res, 1999, 44: $121-131$

4 Sun H, Chartier D, Leblanc N, et al. Intracellular calcium changes and tachycardia-induced contractile dysfunction in canine atrial myocytes. Cardiovasc Res, 2001, 49: 751-761

5 Oh S, Kim K B, Ahn H, et al. Remodeling of ion channel expression in patients with chronic atrial fibrillation and mitral valvular heart disease. Korean J Intern Med, 2010, 25: 377-385

6 Van Wagoner D R, Pond A L, McCarthy P M, et al. Outward $\mathrm{K}^{+}$ current densities and Kv1.5 expression are reduced in chronic human atrial fibrillation. Circ Res, 1997, 80: 772-781

7 Ravens U, Wettwer E. Ultra-rapid delayed rectifier channels: molecular basis and therapeutic implications. Cardiovasc Res, 2011, 89: 776-785

8 Huang B, Chen L, Wu X R, et al. The altered transient outward (Ito1) and ultra-rapid delayed rectifier $\left(I_{\mathrm{Kur}}\right) \mathrm{K}^{+}$currents in right atrial myocytes of human atrial fibrillation. Chin J Pathophysiol, 2005, 21: $77-80$

9 Chiou C W, Eble J N, Zipes D P. Efferent vagal innervation of the canine atria and sinus and atrioventricular nodes: the third fat pad. Circulation, 1997, 95: 2573-2584

10 Liu L, Nattel S. Differing sympathetic and vagal effects on atrial fibrillation in dogs: role of refractoriness heterogeneity. Am J Physiol, 1997, 273: H805-816

11 Ellinor P T, Nam E G, Shea M A, et al. Cardiac sodium channel mutation in atrial fibrillation. Heart Rhythm, 2008, 5: 99-105

12 Balana B, Dobrev D, Wettwer E, et al. Decreased ATP-sensitive K(+) current density during chronic human atrial fibrillation. J Mol Cell Cardiol, 2003, 35: 1399-1405

13 Gaspo R, Bosch R F, Bou-Abboud E, et al. Tachycardia-induced changes in $\mathrm{Na}^{+}$current in a chronic dog model of atrial fibrillation. Circ Res, 1997, 81: 1045-1052

14 Allessie M A, Boyden P A, Camm A J, et al. Pathophysiology and prevention of atrial fibrillation. Circulation, 2001, 103: 769-777

15 Brundel B J, Van Gelder I C, Henning R H, et al. Ion channel remodeling is related to intraoperative atrial effective refractory periods in patients with paroxysmal and persistent atrial fibrillation. Circulation, 2001, 103: 684-690

16 Ausma J, Dispersyn G D, Duimel H, et al. Changes in ultrastructural calcium distribution in goat atria during atrial fibrillation. J Mol Cell Cardiol, 2000, 32: 355-364

17 Grammer J B, Zeng X, Bosch R F, et al. Atrial L-type $\mathrm{Ca}^{2+}$-channel, beta-adrenorecptor, and 5-hydroxytryptamine type 4 receptor mRNAs in human atrial fibrillation. Basic Res Cardiol, 2001, 96: 82-90

18 Greiser M, Halaszovich C R, Frechen D, et al. Pharmacological evidence for altered src kinase regulation of $\mathrm{I}(\mathrm{Ca}, \mathrm{L})$ in patients with chronic atrial fibrillation. Naunyn Schmiedebergs Arch Pharmacol, 2007, 375: 383-392

19 Brundel B J, Van Gelder I C, Henning R H, et al. Alterations in potassium channel gene expression in atria of patients with persistent and paroxysmal atrial fibrillation: differential regulation of protein and mRNA levels for $\mathrm{K}^{+}$channels. J Am Coll Cardiol, 2001, 37: 926-932 
20 Grammer J B, Bosch R F, Kühlkamp V, et al. Molecular remodeling of Kv4.3 potassium channels in human atrial fibrillation. J Cardiovasc Electrophysiol, 2000, 11: 626-633

21 Van Wagoner D R, Pond A L, McCarthy P M, et al. Outward $\mathrm{K}^{+}$current densities and Kv1.5 expression are reduced in chronic human atrial fibrillation. Circ Res, 1997, 80: 772-781

22 Li D, Fareh S, Leung T K, et al. Promotion of atrial fibrillation by heart failure in dogs: atrial remodeling of a different sort. Circulation, 1999, 100: 87-95

23 Xiao H D, Fuchs S, Campbell D J, et al. Mice with cardiac-restricted angiotensin-converting enzyme (ACE) have atrial enlargement, cardiac arrhythmia, and sudden death. Am J Pathol, 2004, 165: 1019-1032

24 Litchenberg W H, Norman L W, Holwell A K, et al. The rate and anisotropy of impulse propagation in the postnatal terminal crest are correlated with remodeling of $\mathrm{Cx} 43$ gap junction pattern. Cardiovasc Res, 2000, 45: 379-387

25 Kostin S, Klein G, Szalay Z, et al. Structural correlate of atrial fibrillation in human patients. Cardiovasc Res, 2002, 54: 361-379

26 Everett T H 4th, Li H, Mangrum J M, et al. Electrical, morphological, and ultrastructural remodeling and reverse remodeling in a canine model of chronic atrial fibrillation. Circulation, 2000, 102: 1454-1460

27 Lewis B P, Shih I H, Jones-Rhoades M W, et al. Prediction of mammalian microRNA targets. Cell, 2003, 115: 787-798

28 Hawkins P G, Morris K V. RNA and transcriptional modulation of gene expression. Cell Cycle, 2008, 7: 602-607

29 Lu Y, Zhang Y, Wang N, et al. MicroRNA-328 contributes to adverse electrical remodeling in atrial fibrillation. Circulation, 2010, 122: 2378-2387

30 Yang B, Lin H, Xiao J, et al. The muscle-specific microRNA miR-1 regulates cardiac arrhythmogenic potential by targeting GJA1 and KCNJ2. Nat Med, 2007, 13: 486-491
31 Girmatsion Z, Biliczki P, Bonauer A, et al. Changes in microRNA-1 expression and IK1 up-regulation in human atrial fibrillation. Heart Rhythm, 2009, 6: 1802-1809

32 Xiao J, Liang D, Zhang Y, et al. MicroRNA expression signature in atrial fibrillation with mitral stenosis. Physiol Genomics, 2011, 43: 655-664

33 Shan H, Zhang Y, Lu Y, et al. Downregulation of miR-133 and miR-590 contributes to nicotine-induced atrial remodelling in canines. Cardiovasc Res, 2009, 83: 465-472

34 van Rooij E, Sutherland L B, Thatcher J E, et al. Dysregulation of microRNAs after myocardial infarction reveals a role of miR-29 in cardiac fibrosis. Proc Natl Acad Sci USA, 2008, 105: 13027-13032

35 Duisters R F, Tijsen A J, Schroen B, et al. miR-133 and miR-30 regulate connective tissue growth factor: implications for a role of microRNAs in myocardial matrix remodeling. Circ Res, 2009, 104: 170-178

36 van Rooij E, Sutherland L B, Qi X, et al. Control of stress-dependent cardiac growth and gene expression by a microRNA. Science, 2007, 316: 575-579

37 Bukowska A, Schild L, Keilhoff G, et al. Mitochondrial dysfunction and redox signaling in atrial tachyarrhythmia. Exp Biol Med, 2008, 233: 558-574

38 Cordes K R, Sheehy N T, White M P, et al. miR-145 and miR-143 regulate smooth muscle cell fate and plasticity. Nature, 2009, 460: 705-710

39 Schlesinger J, Schueler M, Grunert M, et al. The cardiac transcription network modulated by Gata4, Mef2a, Nkx2.5, Srf, histone modifications, and microRNAs. PLoS Genet, 2011, 7: e1001313

40 Ai J, Zhang R, Li Y, et al. Circulating microRNA-1 as a potential novel biomarker for acute myocardial infarction. Biochem Biophys Res Commun, 2010, 391: 73-77

Open Access This article is distributed under the terms of the Creative Commons Attribution License which permits any use, distribution, and reproduction in any medium, provided the original author(s) and source are credited. 